\title{
Fiber Ring Laser Intra-cavity Absorption Spectroscopy for Gas Sensing: Analysis and Experiment
}

\author{
Mo $\mathrm{Li}^{1,2}$, Kun Liu ${ }^{1,3}$, Wencai Jing $^{3}$, and Gang-Ding Peng ${ }^{1 \text { * }}$ \\ ${ }^{1}$ School of Electrical Engineering and Telecommunications, University of New South Wales, \\ Sydney 2052, Australia \\ ${ }^{2}$ Department of Automation Measurement and Control Engineering, Harbin Institute of Technology, \\ Harbin 150001, China \\ ${ }^{3}$ College of Precision Instrument \& Opto-electronics Engineering, Tianjin University, \\ Tianjin 300072, China
}

(Received February 23, 2010 : revised March 8, 2010 : accepted March 8, 2010)

\begin{abstract}
Fiber ring laser based intra-cavity absorption spectroscopic sensor has great potential for high sensitivity gas detection. Using the rate equations and propagation equations, we investigated theoretically factors that affect the sensitivity of such fiber ring laser sensors and determined the optimal design parameters and conditions for significant enhancement of the system sensitivity. Experiments have been conducted to determine the sensitivity enhancement performance. The results showed a factor of $25 \sim 30$ in sensitivity enhancement in the experimental system, agreeing well with the theoretical expectations. Experiments on acetylene detection have also been carried out and the results showed that the ring cavity significantly increases the signal absorption and that high sensitivity can be obtained for gas detection. .
\end{abstract}

Keywords: Fiber ring laser, Intra-cavity absorption, Gas sensor, Sensitivity enhancement

OCIS codes : (060.2370) Fiber optics sensors; (140.3510) Lasers, fiber; (140.3560) Lasers, ring;

(060.2410) Fibers, erbium

\section{INTRODUCTION}

Optical fiber laser gas sensors based on intra-cavity spectroscopy has attracted considerable interest due to their desirable features: inherent fiber compatibility, narrow linewidth, low detection limit, low noise and compact configuration [1]. High sensitivity Inter-Cavity Absorption Gas Sensor (ICAGS) can be achieved by inserting a gas cell into the fiber laser cavity [2]. In an ICAGS, usually Erbium-Doped Fiber (EDF) is used as the gain medium that its broad gain bandwidth covers absorption lines of pollutant gases of interest such as acetylene $\left(\mathrm{C}_{2} \mathrm{H}_{2}\right)$ and permits multi-gas detection without changing light source. Moreover, the EDF based laser source usually exhibits nearly constant output power across a wide tuning range, high signal-to-noise (SNR) ratio, excellent power stability, and high tuning speed for fast modulation and/or detection.

In practical applications, in order to further enhance the sensitivity, other supplemental techniques are often utilized. Zhang et al. applied wavelength modulation technology (WMT) to ICAGS [3]. Ryu et al. measured the pressure broadening coefficients of acetylene molecule based on ICAGS [4]. Liu et al. introduced wavelength sweep technique (WST) to ICAGS and achieved high sensitivity [5].

The performance of an EDF based ICAGS is closely linked to the output signal power, tuning range and sensitivity which are determined by a number of critical parameters: intra-cavity loss, pump power, EDF length, output coupler ratio, etc. Optimal values of these parameters are to be determined, based on appropriate theoretical modeling, for the best system performance. Zhang et al investigated the sensitivity enhancement in EDF based ICAGS using rate equations only [6]. However, the relationship between the ICAGS system sensitivity / performance and the system parameters has not been duly investigated using both rate equations and propagation equations that takes into full

\footnotetext{
*Corresponding author: g.peng@unsw.edu.au

Color versions of one or more of the figures in this paper are available online.
} 
consideration of the dynamics of doped atoms, pump, signal, cavity loss and feedback, etc.

In this paper, we first investigated the sensitivity of ICAGS based on $980 \mathrm{~nm}$ pumped EDF ring laser, taking into consideration of the dynamics of doped atoms (in both ground and excited states), optical pump and signal, cavity loss and feedback, EDF length and gas absorption. Our analysis and simulation are carried out using the coupled rate equations and propagation equations. Firstly the output characteristics of the gas sensing system are briefly discussed. Then the gas detection sensitivity and spectral characteristics of the system are systematically investigated. Moreover, experiments are conducted to examine the sensitivity enhancement factor of the system under different intra-cavity losses and to evaluate ICAGS in acetylene detection. The experimental results agreed well with those of simulations and showed the ICAGS system could realize high sensitivity gas detection.

\section{SYSTEM CONFIGURATION}

The schematic diagram of the gas sensor is shown in Fig. 1, including the following components: a $980 \mathrm{~nm}$ diode pump, a 980/1550 WDM coupler, an EDF, an isolator, a optical circulator, a gas cell, a pigtailed Fabry-Perot tunable filter (TF), a fiber coupler and a tunable attenuator. The isolator and the circulator are used to realize the one-direction transmission of the beam and they help to avoid spatial hole-burning. The bandwidth and the free spectral range (FSR) of the TF are $1.25 \mathrm{GHz}$ and $11.23 \mathrm{THz}$ respectively. The effective length of the gas cell is $10 \mathrm{~cm}$, it is made by aligning a pair of commercial pigtailed selfoc lenses, which enable the gas cell with more favorable performance such as longer working distance and lower insertion losses. The attenuation of the signal induced by the gas is proportional to the gas concentration according to the Lambert-Beer's law, and to be determined by the system.

An EDF fiber laser can be regarded as a two-level system pumped by a $1480 \mathrm{~nm}$ diode laser, or as a three-level system pumped by a $980 \mathrm{~nm}$ laser. When it is pumped by

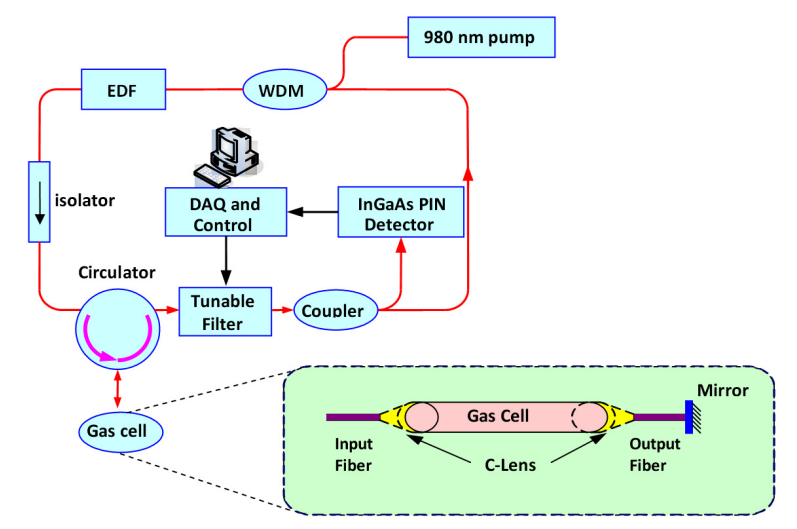

FIG. 1. The configuration of the gas sensor.
$980 \mathrm{~nm}$ diode, the ions absorb the pump power, transit to the high level, stay for a period $\left(\tau_{3}\right)$ and then jump to the metastable state. Due to the value of $\tau_{3}$ is very small, it is reasonable to omit it and simplify the three-level system as two-level system. In our paper, the whole modeling is based on the two-level assumption.

\section{RATE EQUATIONS AND PROPAGATION EQUATIONS}

\subsection{Rate equations}

The rate equations are used to describe the effects of absorption, stimulated emission and spontaneous emission on the ions populations of the ground and metastable states. Based on the homogeneously broadened two-level assumption, the high energy level is assumed to be unpopulated. The atomic population densities of the ground and metastable states, $N_{1}$ and $N_{2}$, over EDF cross section at the ground and metastable energy levels are given by the rate equations $[7,8]$ :

$$
\begin{aligned}
& \frac{d N_{1}(z, t)}{d t}=-\left[W_{s a}(z)+W_{p}(z)\right] N_{1}(z, t)+\left[W_{s e}(z)+1 / \tau_{21}\right] N_{2}(z, t) \\
& \frac{d N_{2}(z, t)}{d t}=-\left[W_{s e}(z)++1 / \tau_{21}\right] N_{2}(z, t)+\left[W_{s a}(z)+W_{p}(z)\right] N_{1}(z, t) \\
& N_{1}(z)+N_{2}(z)=N_{0}
\end{aligned}
$$

where $\tau_{21}$ is the spontaneous emission lifetime, $N_{0}$ the dopant density of the EDF, $W_{s a}, W_{s e}$ and $W_{p}$ are the stimulated absorption, stimulated emission and pumping rates, respectively, which can be described by [9-10]:

$$
\begin{aligned}
W_{s a}(z)= & \frac{1}{A_{e f f}} \int_{0}^{\infty} \frac{\sigma_{a}(v)}{h v}\left[P_{A S E^{+}}(z, v)+P_{A S E^{-}}(z, v)\right] \eta(v) d v \\
= & \sum_{v} \frac{\sigma_{a}(v)}{A_{e f f} h v}\left[P_{A S E^{+}}(z, v)+P_{A S E^{-}}(z, v)\right] \eta(v) \\
W_{s e}(z)= & \sigma_{e}\left(v_{p}\right) \frac{P_{p}^{+}(z)+P_{p}^{-}(z)}{h v_{p} A_{e f f}} \eta\left(v_{p}\right)+\frac{1}{A_{e f f}} \int_{0}^{\infty} \frac{\sigma_{e}(v)}{h v} \\
& {\left[P_{A S E^{+}}(z, v)+P_{A S E^{-}}(z, v)\right] \eta(v) d v } \\
= & \sigma_{e}\left(v_{p}\right) \frac{P_{p}^{+}(z)+P_{p}^{-}(z)}{h v_{p} A_{e f f}} \eta\left(v_{p}\right)+\sum_{v} \frac{\sigma_{e}(v)}{A_{e f f} h v} \\
& {\left[P_{A S E^{+}}(z, v)+P_{A S E^{-}}(z, v)\right] \eta(v) } \\
W_{p}(z)= & \sigma_{a}\left(v_{p}\right) \frac{P_{p}^{+}(z)+P_{p}^{-}(z)}{h v_{p} A_{e f f}} \eta_{p}
\end{aligned}
$$

In Eq.4-6, $\sigma_{a}$ is the absorption cross-section of EDF, $\sigma_{e}$ is the emission cross-section of EDF, $h$ is Plank's constant, $P_{p}^{+}(z), P_{p}^{-}(z), P_{A S E^{*}}(z)$ and $P_{A S E^{-}}(z)$ are the co-propagating and counter-propagating pump powers and ASE powers at 
frequency $\nu$ with a frequency interval $\Delta \nu$ at a longitudinal fiber coordinate $z, \nu_{p}$ is the frequency of the pump. $A_{\text {eff }}$ is the effective fiber core area and $\eta(\nu)$ is the overlap integral between the $L P_{01}$ mode intensity distribution $\mathrm{E}(r, \nu)$ and the erbium doping density function $\rho(r)$ which can be given by the following equation, where $r$ is the radius of the EDF:

$$
\eta(v)=\frac{\int_{0}^{\infty} \rho(r)|E(r, v)|^{2} r d r}{\int_{0}^{\infty}|E(r, v)|^{2} r d r}
$$

The steady state photon number on the two-level can be obtained from Eq.1-3 by setting $\frac{d N_{1}(z, t)}{d t}=0$ and $\frac{d N_{2}(z, t)}{d t}=0$, we can get:

$$
\begin{aligned}
& N_{1}(z)=\frac{W_{s e}(z)+1 / \tau_{21}}{W_{s a}(z)+W_{s e}(z)+W_{p}(z)+1 / \tau_{21}} N_{0} \\
& N_{2}(z)=\frac{W_{s a}(z)+W_{p}(z)}{W_{s a}(z)+W_{s e}(z)+W_{p}(z)+1 / \tau_{21}} N_{0}
\end{aligned}
$$

In combination with Eq.4-7, we can get expression of $N_{1}$ and $N_{2}$.

\subsection{Propagation equations}

We can assume light in the EDF amplifier propagates as a number of optical beams of frequency bandwidth $\Delta \nu$ centered at the wavelength $\lambda=c / \nu$. All emission and absorption cross sections included in the model are spectrally resolved. The spectral region from 1480 to 1600 $\mathrm{nm}$ is subdivided into 700 slots with $\Delta \lambda=0.2 \mathrm{~nm}$.

The propagation equation of the pump power $P_{p}^{ \pm}(z)$ and ASE power $P_{A S E^{ \pm}}(z, v)$ is described by the following propagation equations [11-13]:

$$
\begin{aligned}
& \frac{d P_{p}^{ \pm}(z)}{d z}=\mp\left[g_{a}\left(z, v_{p}\right)-g_{e}\left(z, v_{p}\right)\right] P_{p}^{ \pm}(z) \\
& \frac{d P_{A S E^{ \pm}}(z, v)}{d z}= \pm 2 h v \Delta v g_{e}(z, v) \pm\left[g_{e}(z, v)-g_{a}(z, v)\right] P_{A S E^{ \pm}}(z, v)
\end{aligned}
$$

where $g_{e}(z, \nu)$ and $g_{a}(z, \nu)$ are the emission and absorption factors given by Eq.12 and 13. The term $2 h \nu$ $\Delta \nu g_{e}(z, \nu)$ is the ASE power induced by the spontaneous emission.

$$
\begin{aligned}
& g_{e}(z, v)=\eta(v) \sigma_{e}(v) N_{2}(z) \\
& g_{a}(z, v)=\eta(v) \sigma_{a}(v) N_{1}(z)
\end{aligned}
$$

Due to the isolator and single pump we use in the system, the pump and ASE light can be assumed to only transmit in the forward direction as follows (when the pump wavelength is $\left.980 \mathrm{~nm}, \sigma_{e}\left(\nu_{p}\right)=0\right)$ :

$$
\begin{aligned}
& \frac{d P_{p}(z)}{d z}=-\sigma_{a}\left(v_{p}\right) N_{1}(z) \eta\left(v_{p}\right) P_{p}(z) \\
& \begin{aligned}
\frac{d P_{A S E}(z, v)}{d z}= & 2 h v \Delta v \sigma_{e}(v) N_{2}(z) \eta(v)+\mid \\
\cdot & {\left[\sigma_{e}(v) N_{2}(z)-\sigma_{a}(v) N_{1}(z)\right] \eta(v) P_{A S E}(z, v) }
\end{aligned}
\end{aligned}
$$

In order to model the ring laser operation, the output signals of the EDF amplifier are carried back to the input. Initially, the ASE power generated during the first step becomes the input signal of the second run and the signal obtained at the end of EDF is amplified in each run.

An iterative solution of the rate equations and propagation equations for the pump and both the forward and backward propagating amplified spontaneous emission powers was implemented using a fourth-order Runge-Kutta routine. Convergence can be reached in a few iterations. Based on the configuration shown in Fig. 1, appropriate boundary conditions are imposed at the beginning and at the end of the active fiber $(z=0, L)$ on the pump power and the individual spectral components of forward and backward ASE and pump powers generated in the EDF. The powers are filtered by the tunable filter and attenuated by the loss of the intra-cavity and part of it is output via a 50:50 coupler. The boundary conditions for the pump and ASE powers are defined as follows:

$$
\begin{aligned}
& P_{p}^{+}(z=0)=P_{p 0} \\
& P_{A S E}^{k}(z=0, v)=P_{A S E}^{k-1}(z=L, v) F(v) \delta_{\text {ring }}\left(1-\alpha_{\text {coup }}\right)
\end{aligned}
$$

where $P_{p 0}$ is the initial pump power coupled into the $\mathrm{EDF}$, the upper index $k$ denotes the iteration number, $\delta_{\text {ring }}$ represents the total loss of the cavity, the coupler and the losses of other optical components. $\alpha_{\text {coup }}$ is the ratio of the coupler and $\mathrm{F}(\nu)$ expressed in Eq.17 is the relative transmittance of the optical band-pass filter.

$$
F(\lambda)=T_{j F} \exp \left[-\frac{4 \ln 2\left(\lambda-\lambda_{j F}\right)^{2}}{\sigma_{j F}^{2}}\right]
$$

In the above equation, $T_{j F}$ is the peak transmittance of the tunable filter, $\sigma_{j F}$ is the bandwidth of the transmission spectrum. $\lambda_{j F}$ is the central wavelength of the tunable filter.

\subsection{Sensitivity analysis}

The absorption spectroscopy is described based on Lambert-Beer's law which is given as below [14]: 


$$
I(v)=I_{0}(v) \exp [-k(v) L]
$$

where $I$ and $I_{0}$ are the laser output power with and without gas in the cavity respectively. We define the absorption signal $k$ as $k=\operatorname{In}\left(I_{0} / I\right)$. Noting that $\Delta I=I_{0}-I$ denotes the reduction of the output power due to the cavity loss $\Delta \delta$ induced by the gas absorption. For a small gas absorption, $\mathrm{k} \approx \Delta I / I$. The sensitivity enhancement factor $K$ can be defined as the ratio of the intra-cavity absorption signal $k$ over the single pass loss $\Delta \delta$ [15]:

$$
K=\frac{k}{\Delta \delta}=\frac{\Delta I}{I \Delta \delta}
$$

\section{THEORETICAL AND EXPERIMENTAL STUDY}

\subsection{Theoretical results and discussions}

\subsubsection{Pump threshold}

An EDF from Nufern is to be used in our experiment. Its absorption/emission coefficients are $23 \mathrm{~dB} / \mathrm{m}$ and 24 $\mathrm{dB} / \mathrm{m}$ respectively. The dopant concentration of the EDF is $6.8 \times 10^{24}$ ions $/ \mathrm{m}^{3}$, the core diameter is $3.2 \mu \mathrm{m}$ and the numerical aperture is 0.25 . The absorption and stimulated emission coefficient and overlap integral between the optical mode and the erbium ions of the EDF are shown in Fig. 2. From the simulations we found that the pump threshold is closely related to the difference between the absorption cross-section and emission cross-section. The overlap integral and the dopant concentration also affect the value of the pump threshold.

The TF we use in experiment is from Micron Optics with a bandwidth of $10 \mathrm{pm}$. The peak transmission of the $\mathrm{TF}$ is about 0.8 . Other parameters we used which we don't specify can be found in Table.1.

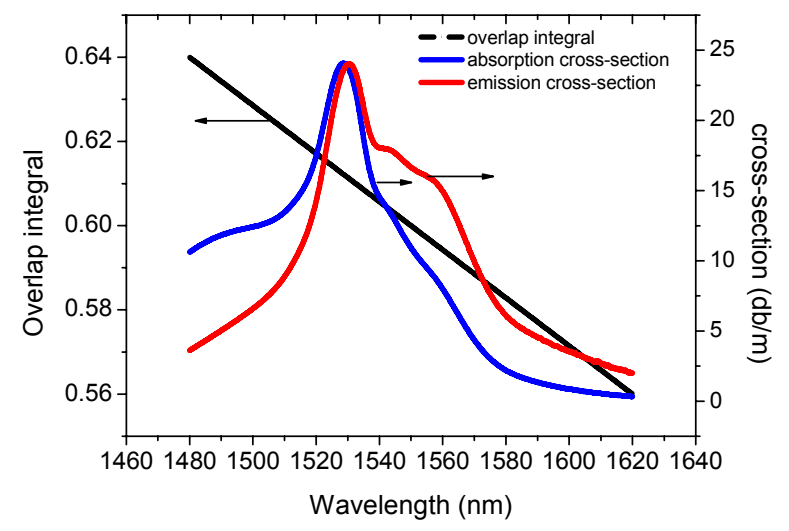

FIG. 2. The emission/absorption coefficient and the overlap integral between the $\mathrm{LP}_{01}$ mode intensity distribution and the erbium ion doping density function of the EDF.
Using the coupled rate equations and propagation equations established in the previous section and the parameters given above, in the following we will first investigate the pump threshold characteristics of the gas sensing system and then present a detailed investigation of the gas detection sensitivity and spectral characteristics of the system.

The effects of length of EDF and intra-cavity loss on the pump threshold have been determined and shown in Fig. 3. In Fig. 3 (a), the cavity loss is set at $10 \mathrm{~dB}$ and

Table. 1 Parameters used in numerical simulations

\begin{tabular}{c|c}
\hline \hline Dopant concentration & $6.8 \times 10^{24} \mathrm{~m}^{-3}$ \\
\hline Fiber core diameter & $3.2 \times 10^{-6} \mathrm{~m}$ \\
\hline Pump wavelength & $980 \mathrm{~nm}$ \\
\hline Signal wavelength & $1530.4 \mathrm{~nm}$ \\
\hline Life time of the stable level & $10 \mathrm{~ms}$ \\
\hline Coupler ratio & $50: 50$ \\
\hline WDM ratio & $9: 1$ \\
\hline $\begin{array}{c}\text { Absorption coefficient of } \mathrm{C}_{2} \mathrm{H}_{2} \\
\text { at signal wavelength }\end{array}$ & $\begin{array}{c}1.21 \times 10^{-20} \\
\mathrm{~cm}^{-1} /\left(\mathrm{molecule} \cdot \mathrm{cm}^{-3)}\right.\end{array}$ \\
\hline \begin{tabular}{c} 
Optical path length of the absorber \\
\hline
\end{tabular} & $10 \mathrm{~cm}$ \\
\hline
\end{tabular}

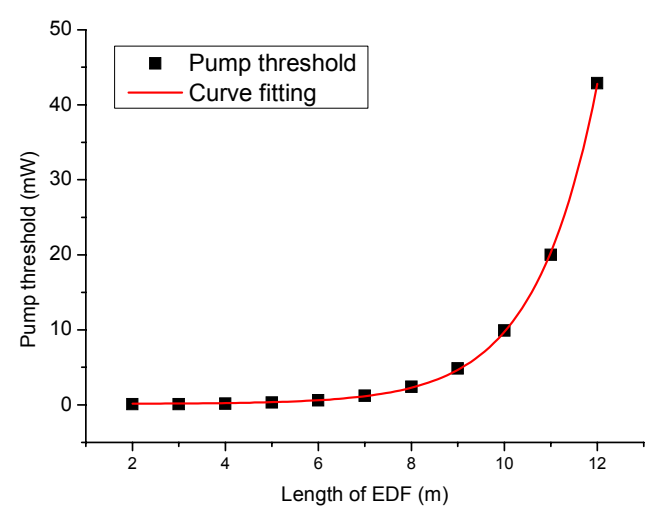

(a)

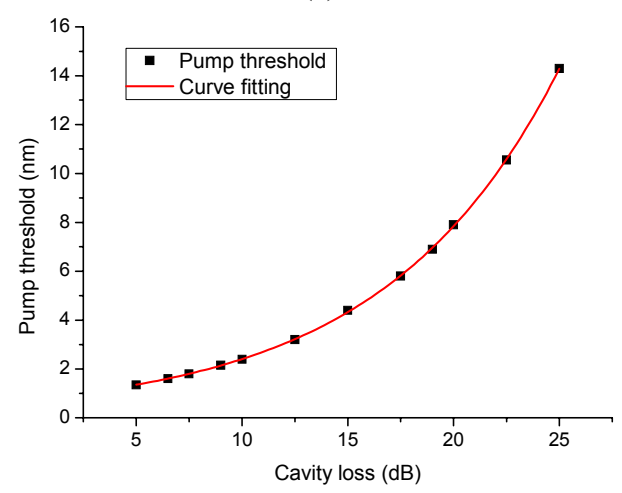

(b)

FIG. 3. (a) The relationship between the length of EDF and the pump threshold; (b) The relationship between the intra-cavity loss and the pump threshold. 
the solid line is the fitted curve of the simulated results. It is obvious that the threshold pump changes in exponential relationship with the length of the EDF. In Fig. 3 (b), the pump power is set at $2.4 \mathrm{~mW}$, and the fitted curve also shows an exponential profile. From the results, we can see that, the pump threshold goes up with the increase of the EDF length and intra-cavity loss.

\subsubsection{Sensitivity enhancement}

In Fig. 4 we show the theoretical results of the influences of pump power, cavity loss and EDF length on the sensitivity enhancement factor.

From Fig. 4 (a) and (b), we can find that the highest sensitivity is achieved at the pump threshold and the cavity loss threshold. Increasing the pump power and decreasing the cavity loss have the same effects on the sensitivity enhancement factor of the gas sensor. Higher cavity loss means higher pump threshold and higher pump power means lower cavity loss threshold when the EDF length is constant. We can also find that, the value of the peak sensitivity varies with the pump power and cavity loss. In Fig. 4 (a), among four different cavity losses, the highest sensitivity can be obtained when the cavity loss is $10 \mathrm{~dB}$ and the pump power is at its threshold of $2.4 \mathrm{~mW}$. In Fig. 4 (b), for four different pump powers, the highest sensitivity can be achieved when pump power is $2.4 \mathrm{~mW}$ and the cavity loss is at its threshold of $10 \mathrm{~dB}$. Due to the laser is usually not stable working right at the threshold, in practical application, we should make the system running a little beyond the threshold to have both high sensitivity and high stability.

In Fig. 4 (c), when the intra-cavity loss is $10 \mathrm{~dB}$, the system reaches its peak sensitivity with different EDF lengths and at different pump powers. Higher pump power leads to shorter EDF length required to achieve its peak sensitivity. In addition, higher pump power also results in higher peak sensitivity enhancement factor when the intra-cavity loss is constant. Among the four pump powers we considered, the greatest peak sensitivity is obtained when pump power is $7.9 \mathrm{~mW}$. In Fig. 4 (d), when the pump power is $2.4 \mathrm{~mW}$, the optimal EDF length varies with different intra-cavity losses. We can conclude that the higher the cavity loss is, the lower the peak sensitivity will be. If the cavity loss is decreased under the same pump power, a longer EDF is required to achieve the peak sensitivity. It is clear that the optimal lengths of EDF under different pump power and cavity loss need to be investigated before conducting experiments.

In order to establish the relationship between the pump power and the sensitivity enhancement factor with regard to the pump threshold, we introduce the ratio of pump power over the pump threshold in Fig. 5. It is obvious that the sensitivity varies with the ratio and reaches its maximum exactly at 1 . This means that the peak sensitivity enhancement is always peaked at the pump threshold, regardless the values of intra-cavity loss, although the magnitude

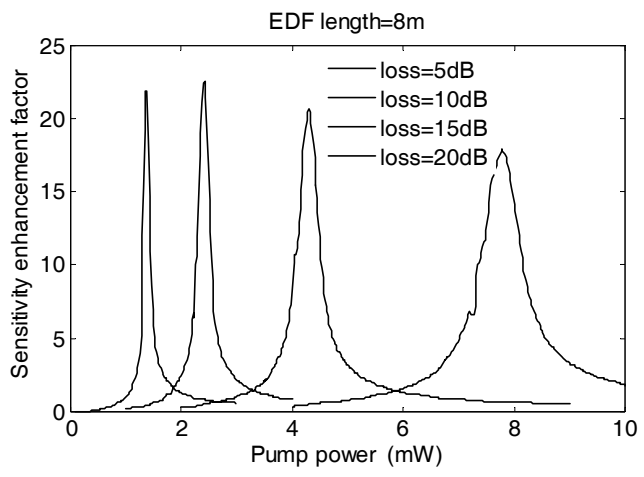

(a)

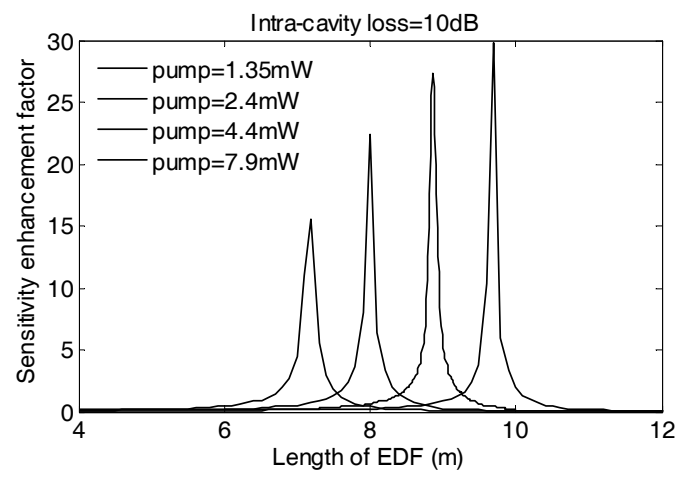

(c)

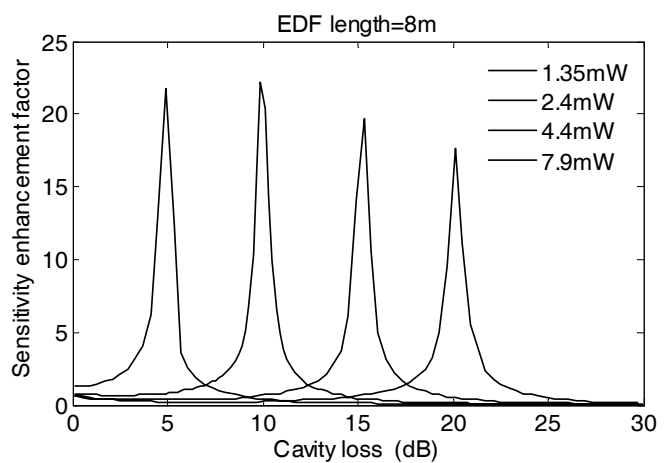

(b)

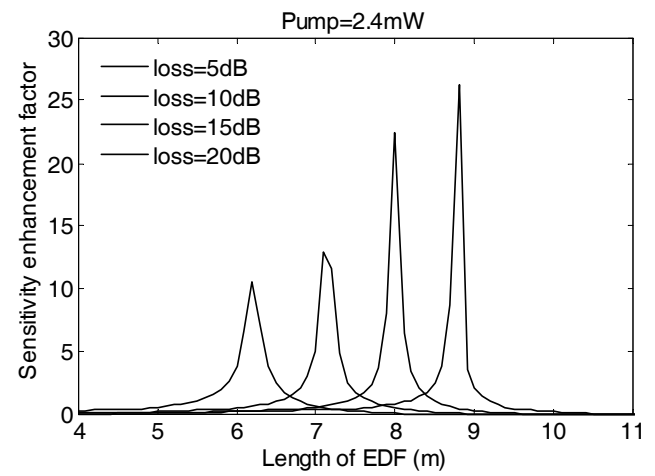

(d)

FIG. 4. The influences of pump power, cavity loss and EDF length on sensitivity enhancement factor. 


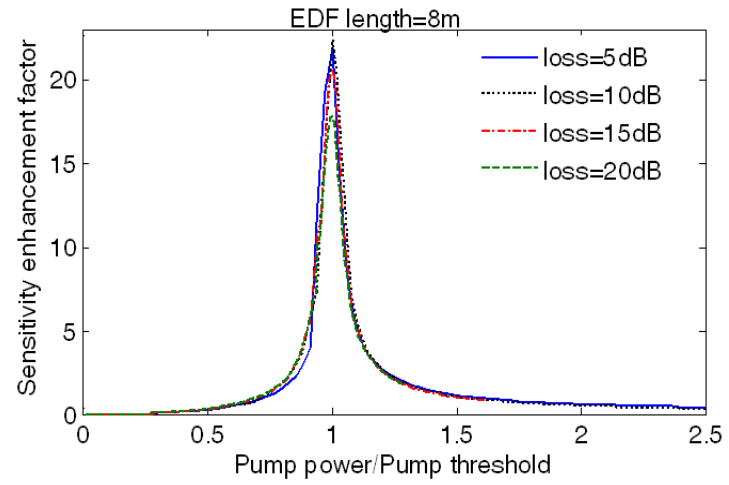

FIG. 5. The relationship between the ratio of pump power over pump threshold and sensitivity enhancement factor under different cavity losses.

of the peak sensitivity changes with the value of intra-cavity losses.

\subsubsection{Spectral characteristics}

Fig. 6 shows the output power and tuning (operating) range of the system as a function of wavelength of 8-meter-long EDF at $100 \mathrm{~mW}$ pump power, with four intra-cavity losses: $5 \mathrm{~dB}, 10 \mathrm{~dB}, 15 \mathrm{~dB}$ and $20 \mathrm{~dB}$ respectively. It is obvious that the output power and tuning wavelength range are very sensitive to the intra-cavity loss. Both the output power and the tuning range increase significantly if the intra-cavity loss reduces. When the intra-cavity loss is high, e.g. $20 \mathrm{~dB}$, the tuning bandwidth covers $\mathrm{C}$ band only. However, with a lower intra-cavity loss, e.g. $5 \mathrm{~dB}$, a much larger tuning wavelength band $(\mathrm{C}+\mathrm{L})$ can be obtained. It is clear that tuning range is more sensitive to intra-cavity loss at the long wavelength side than at the short wavelength side. When the cavity loss increases from $5 \mathrm{~dB}$ to $15 \mathrm{~dB}$, the range at the long wavelength side decreases nearly 40 $\mathrm{nm}$, while at the short wavelength side decreases less than $10 \mathrm{~nm}$. These results reveal that low intra-cavity loss is necessary if large tuning wavelength range and high output power are desired.

To investigate the effect of EDF length on the tuning wavelength range and the output power, we set the intra-cavity loss at $10 \mathrm{~dB}$ and the pump power at $100 \mathrm{~mW}$ for five EDF lengths $(1.5 \mathrm{~m}, 4 \mathrm{~m}, 8 \mathrm{~m}, 15 \mathrm{~m}$ and $25 \mathrm{~m})$. The simulation result is shown in Fig. 7. It can be seen that, for the EDF length equals $1.5 \mathrm{~m}$, the tuning bandwidth is narrow (about $40 \mathrm{~nm}$ ) and the center wavelength is near $1540 \mathrm{~nm}$. With an increase of EDF length (from 4 $\mathrm{m}$ to $8 \mathrm{~m}$ ), the tuning bandwidth becomes broader and the center wavelength shifts to the longer wavelength band, the output power also increase a little. With the EDF length longer than $15 \mathrm{~m}$, the tuning range and output power reach their maximum and become quite stable.

The effect of cavity loss on the tuning (operating) range of the system is analyzed for a piece of $8 \mathrm{~m}$ EDF with pump power of $100 \mathrm{~mW}$ and $150 \mathrm{~mW}$ respectively. We

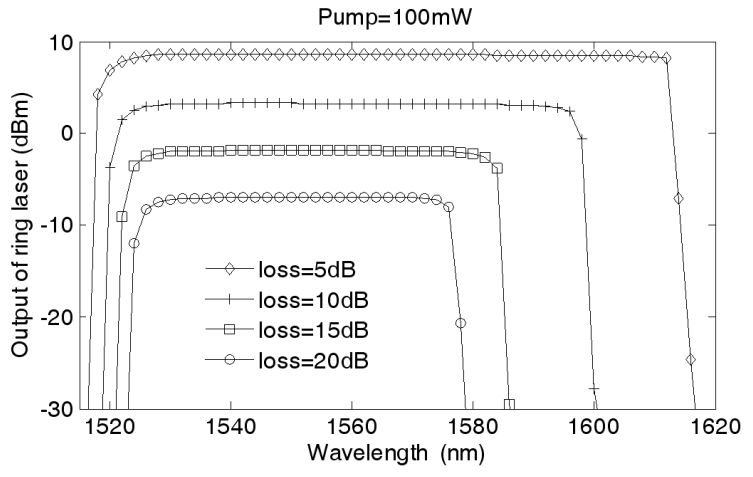

FIG. 6. Output signal power under different intra-cavity loss at pump power of $100 \mathrm{~mW}$.

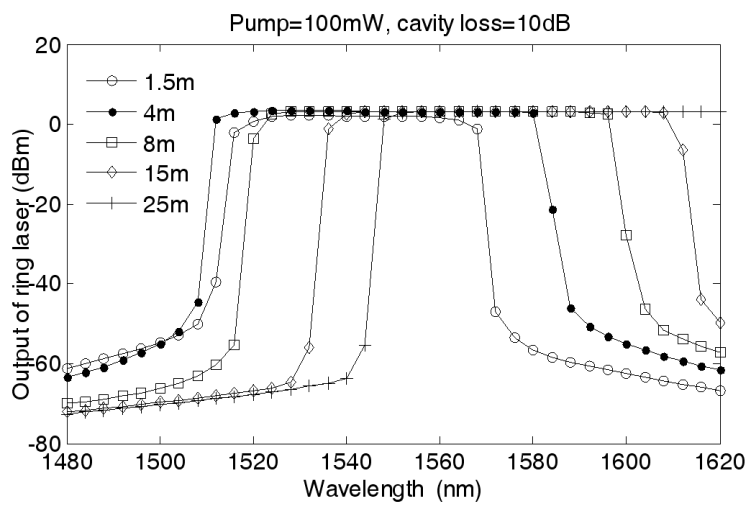

FIG. 7. Output signal power vs wavelength with varying EDF lengths.

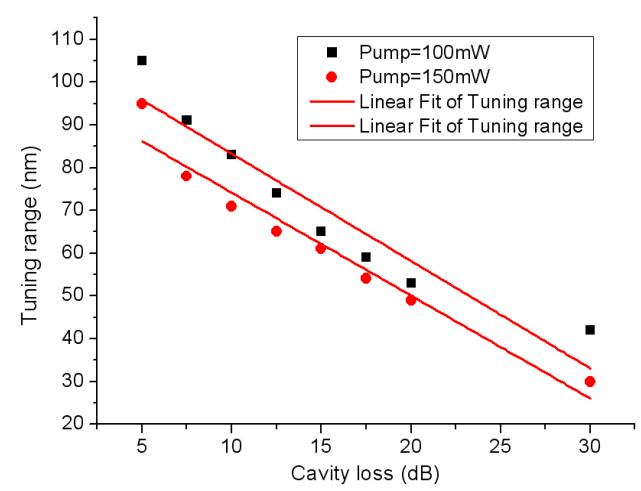

FIG. 8. The relationship between the tuning (operating) range and the cavity loss.

show the results and the fitted curves of the results in Fig. 8. The tuning range decreases nearly linearly with the increase of the cavity loss if the pump power is fixed. When the cavity loss is the same, higher pump power means wider tuning range. From the figure, we can safely conclude that increasing the pump power and decreasing the cavity loss are two effective methods to make the tuning range of the system broader. 


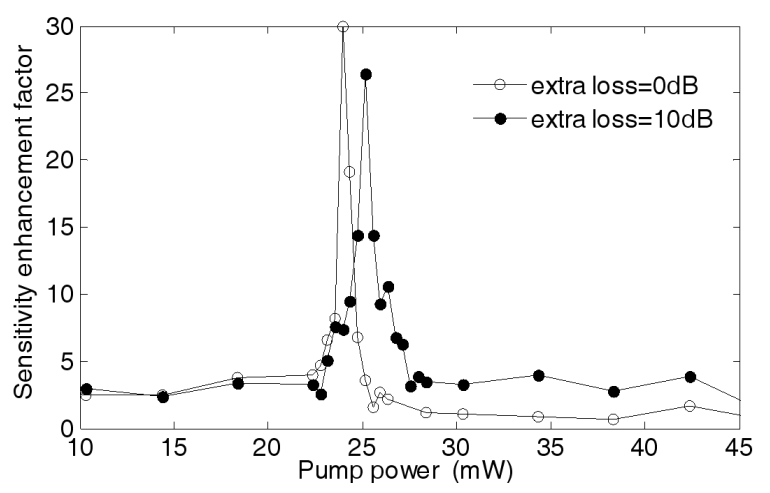

FIG. 9. The measured sensitivity enhancement factor of the system with different cavity losses.

\subsection{Experimental results and discussions}

\subsubsection{Sensitivity enhancement}

The sensitivity enhancement factor can be tested by experiments. Measurements are conducted using a similar system as shown in Fig. 1. The dependence of the sensitivity enhancement factor on the pump power is measured and shown in Fig. 9. The EDF we use in the experiment is different with the one we use in the simulation. The experimental EDF has a length of $2.5 \mathrm{~m}$. The intra-cavity loss is about $10 \mathrm{~dB}$. The measurement is conducted twice, without and with an extra loss equals $10 \mathrm{~dB}$. The amplitude of the output power was collected for 15 times and then averaged, with $\Delta \delta=0.1 \mathrm{~dB}$ (as defined in Eq.19). It is obvious that the experimental results are in accordance with the theoretical expectation. The highest sensitivity can be obtained at the pump threshold. The greater intra-cavity loss leads to the higher pump threshold. The peak sensitivity of the system without extra loss is higher than that with extra loss.

\subsubsection{Gas detection with ICAGS}

Gas sensing experiments are also conducted with the system setup shown in Fig. 1. The resolution of the tunable attenuator is $0.01 \mathrm{~dB}$. The gas cell is made by aligning a pair of commercial pigtailed selfoc lenses. This design of the gas cell could have long path length and low insertion loss. The length of the gas cell is about $10 \mathrm{~cm}$. The parameters of the TF are the same with that we use in the theoretical analysis.

There are three strong absorption lines of acetylene near $1530 \mathrm{~nm}$ and we define they are Spectrum1, Spectrum 2, Spectrum 3 which are at $1531.588 \mathrm{~nm}, 1530.371 \mathrm{~nm}$ and $1529.180 \mathrm{~nm}$ respectively [5]. From the HITRAN database, we can get the absorption cross-sections of the three absorption lines are $1.17 \times 10^{-20} \mathrm{~cm}^{-1} /\left(\right.$ molecule $\left.\cdot \mathrm{cm}^{-3}\right), 1.21 \times 10^{-20} \mathrm{~cm}^{-1} /$ $\left(\right.$ molecule $\left.\cdot \mathrm{cm}^{-3}\right), 1.14 \times 10^{-20} \mathrm{~cm}-1 /\left(\right.$ molecule $\left.\cdot \mathrm{cm}^{-3}\right)$ [16]. By adjusting the voltage applied on the TF, we can scan the three absorption line in $90 \mathrm{~s}$ and get the average value.

The experimental data we got were scattered data requiring

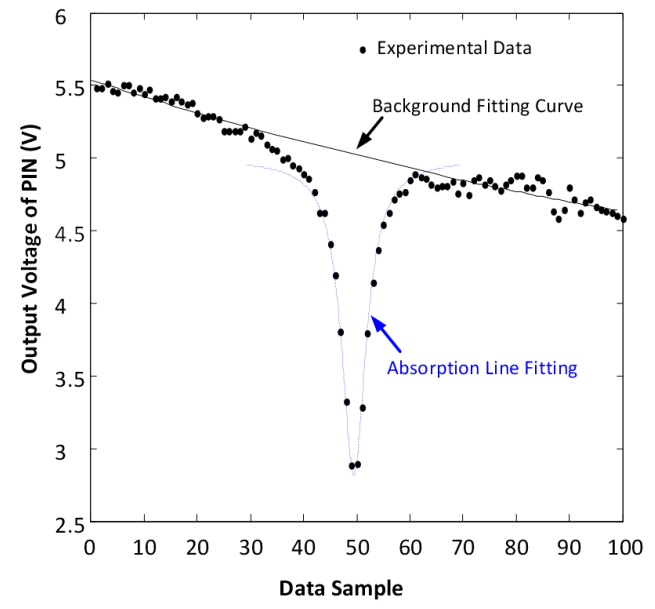

FIG. 10. The attenuation of the laser power induced by the gas using the curve-fitting method.

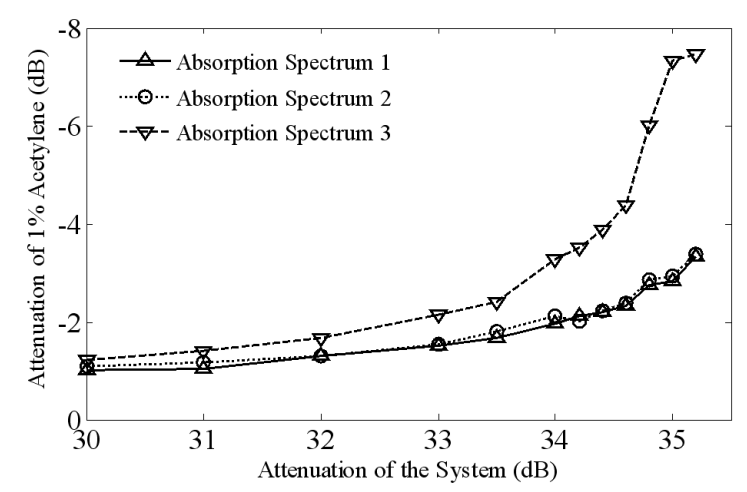

FIG. 11. The relationship between the absorption signal and the system attenuation.

further processing to get the exact position of the absorption peak and calculate the attenuation induced by the gas absorption. Under room temperature and normal pressure, the absorption line shape is Lorentzian profile. With the data processing method shown in Fig. 10, the experimental data can be curve-fitted by Lorentzian function and the position of the peak of the Lorentzian profile is used as the position of the absorption line.

The system can be used to realize gas detection and the amplitude of the absorption signal is proportional to the concentration of the gas. When the gas cell was filled with $1 \%$ acetylene and the pump driving current was set to 80 $\mathrm{mA}$, the relationship between the absorption signal and the system attenuation is shown in Fig. 11. Of the three absorption lines, the strongest absorption happens at 1529.180 $\mathrm{nm}$, and the attenuation of the other two absorption signals is similar. For the strongest absorption line, the maximum of the absorption signal was about $7.5 \mathrm{~dB}$, corresponding to an enhancement of more than 30 times over the singlepass absorption loss of only $0.2 \mathrm{~dB}$. This means that the sensitivity is greatly enhanced in our ICAGS system. 


\section{CONCLUSIONS}

We have theoretically and experimentally studied the operation and performance of fiber ring laser based ICAGS, using a $980 \mathrm{~nm}$ diode pumped EDF. Our theoretical analysis and simulation are carried out using the coupled rate equations and propagation equations, taking into consideration of the dynamics of doped atoms (in both ground and excited states), optical pump and signal, cavity loss and feedback, EDF length and gas absorption. The sensitivity enhancement and spectral characteristics of the system are systematically investigated with respect to the pump power, the intra-cavity loss and the EDF length. From our theoretical analysis, we draw the following conclusions: The peak sensitivity (enhancement) is reached exactly at the pump threshold or the cavity loss threshold; The peak sensitivity varies with both the pump power and cavity loss, i.e. for fixed values of the pump power and cavity loss, we can find an optimal EDF length and achieve a peak sensitivity; With a fixed value of the pump power, both the optimal EDF length and the peak sensitivity decrease with the increase of cavity loss; With a fixed value of the intra-cavity loss, both the optimal EDF length and the peak sensitivity increase with the increase of cavity loss; Both the tuning (or operating) range and output signal power are sensitive to the pump power and cavity loss. Higher pump power and lower intracavity loss would have broader tuning range and greater output signal power; The EDF length also affects the tuning range and output signal power, i.e. with the increase of EDF length, the tuning range and output power increase.

The experiment to measure the sensitivity of ICAGS is conducted and the results agree well with the theoretical predictions. Using WST, real time gas sensing is realized with the ICAGS and $1 \%$ acetylene is tested. In our experimental ICAGS, the signal absorption is significantly enhanced in comparison to that of the single-pass absorption gas sensor, thus demonstrating that the ICAGS system could realize high sensitivity gas detection.

\section{ACKNOWLEDGMENT}

Authors acknowledge funding of an International Science Linkages (ISL) Australia China Special Fund project (CH060036) from the Department of Industry, Innovation, Science and Research (DIISR), Australia.

\section{REFERENCES}

1. G. Stewart, K Atherton, H. B. Yu, and B. Culshaw, "An investigation of an optical fibre amplifier loop for intra-cavity and ring-down cavity loss measurements," Meas. Sci. Technol. 12, 843-849 (2001).

2. Y. Zhang, M. Zhang, and W. Jin, "Multi-point, fiber-optic gas detection with intra-cavity spectroscopy," Opt. Comm. 220, 361-364 (2003).

3. M. Zhang, D. N. Wang, W. Jin, and Y. B. Liao, "Wavelength modulation technique for intra-cavity absorption gas sensor," IEEE Trans. Instrum. Meas. 53, 136-139 (2004).

4. H. Y. Ryu, W. K. Lee, H. S. Moon, and H. S. Suh, "Tunable Erbium-doped fiber ring laser for applications of infrared absorption spectroscopy," Opt. Comm. 275, 379-384 (2007).

5. K. Liu, W. C. Jing, G. D. Peng, J. Z. Zhang, Y. Wang, T. G. Liu, D. Jia, H. Zhang, and Y. Zhang, "Wavelength sweep of intra-cavity fiber laser for low concentration gas detection," IEEE Photon. Technol. Lett. 20, 1515-1517 (2008).

6. Y. Zhang, M. Zhang, and W. Jin, "Sensitivity enhancement in Erbiumdoped fiber laser intra-cavity absorption sensor," Sens. Actuators A 104, 183-187 (2003).

7. X. Dong, P. Shum, N. Q. Ngo, H.-Y. Tam, and X. Dong, "Output power characteristics of tunable Erbium-doped fiber ring lasers," IEEE J. Lightwave Technol. 23, 1334-1341 (2005).

8. A. Bellemare. M. Karbsek, C. Riviere, F. Babin, G. He, V. Roy, and G. W. Schinn, "A broadly tunable Erbium-doped fiber ring laser: experimentation and modeling," IEEE. J. Select. Topics Quantum Electron. 7, 22-29 (2001).

9. T. Pfeiffer, H. Schmuck, and H. Bulow, "Output power characterisitics of Erbium-doped fiber ring lasers," IEEE Photon. Technol. Lett. 4, 847-849 (1992).

10. S. Selvakennedy, M. A. Mahdi, M. K. Abdullah, P. Poopalan, and H. Ahmad, "Design optimisation of Erbium-doped fiber ring laser through numerical simulation," Opt. Comm. 170, 247-253 (1999).

11. S. Selvakennedy, M. A. Mahdi, M. K. Abdullah, P. Poopalan, and H. Ahmad, "Behavioral investigaions of an Erbiumdoped fiber ring laser through numerical simulations," Opt. Fiber Technol. 6, 155-163 (2000).

12. M. Karasek and J. A. Valles, "Analysis of channel addition/ removal response in all-optical gain-controlled cascade of Erbium-doped fiber amplifiers," IEEE J. Lightwave Technol. 16, 1795-1803 (1998).

13. C. R. Giles and E. Desurvire, "Modeling Erbium-doped fiber amplifiers," IEEE J. Lightwave Technol. 9, 271-283 (1991).

14. V. M. Baev, T. Latz, and P. E. Toschek, "Laser intracavity absorption spectroscopy," Appl. Phys. B 69, 171-202 (1999).

15. Y. Zhang, M. Zhang, W. Jin, H. L. Ho, M. S. Demokan, X. H. Fang, B. Culshaw, and G. Stewart, "Investigation of Erbium-doped fiber laser intracavity absorption sensor for gas detection," Opt. Comm. 234, 435-441 (2004).

16. HITRAN Molecular Spectroscopic Database 2004. 